\title{
Morphological variation in Blommersia pelvic shape coincides with muscular architecture and parallels genus phylogenetic evolution
}

\author{
Javier H. Santos-Santos ${ }^{1,2,}$, Mireia Guinovart-Castán ${ }^{1}$, David R. Vieites ${ }^{1}$ \\ ${ }^{1}$ Department of Biogeography and Global Change (BGC-MNCN-CSIC), Museo Nacional de Ciencias \\ Naturales, C/José Gutiérrez Abascal 2, E-28006, Madrid, Spain. \\ ${ }^{2}$ Department of Animal Biology, University of Barcelona, Avenida Diagonal 645, 08028, Barcelona, Spain. \\ *Email: jssantos@usal.es
}

"This manuscript has been withdrawn by the authors as it was submitted without appropriate permission. If you have any questions, please contact the corresponding author." 